\title{
Pé Diabético e Doença Vascular - Entre o Conhecimento Acadêmico e a Realidade Clínica
}

\begin{abstract}
$\triangle$ população diabética no mundo está em franca expansão. Estima-se que o número de pacientes salte dos quase 200 milhões atuais para mais de 300 milhões de indivíduos nos próximos 20 anos (1). Na última década experimentamos grandes avanços na compreensão da fisiopatologia, na abordagem e no tratamento do diabetes e de suas complicações. Ainda hoje, no entanto, a doença vascular associada ao diabetes suscita dúvidas conceituais e questionamentos sobre o papel das alterações das macro e microcirculações na gênese e no desfecho das complicações comuns desse paciente.

O diabetes melito é a principal causa de cegueira, doença renal terminal e amputação de membros na população norte-americana. Um indivíduo diabético tem entre 15 e 40 vezes mais chance do que a população geral de submeter-se a uma amputação do membro inferior. Em pacientes com lesões infectadas e isquêmicas, o risco pode ser 90 vezes maior comparado ao apresentado por pacientes sem isquemia ou infecção.
\end{abstract}

A hiperglicemia sustentada ao longo do tempo determina uma série de alterações estruturais e bioquímicas em órgãos-alvo, especialmente em olhos, rins, coração, artérias e nervos periféricos. A duração e a magnitude da hiperglicemia são determinantes da velocidade de progressão da doença microvascular. As células endoteliais não são capazes de controlar o transporte de glicose e sofrem danos iniciados com a manutenção da hiperglicemia intracelular. No endotélio disfuncional, os efeitos vasoconstritores se sobrepõem aos efeitos vasodilatadores, ocorre perda do controle vasomotor, espessamento do endotélio vascular e alteração da relação endotélio-célula sanguínea, especialmente plaquetas e leucócitos.

$\mathrm{O}$ artigo de Santos e cols. (2) discute alguns aspectos interessantes da doença vascular por meio do estudo da histologia das macro e microcirculações em pacientes submetidos à amputação de membros. A microcirculação é tema de intensa discussão e especulação. O espessamento do endotélio vascular como padrão da microangiopatia diabética é controverso e encontra na literatura defensores e algozes. Mesmo quando os exames histológicos confirmam as alterações da parede do vaso, há diferentes padrões descritos, não exclusivos do leito vascular diabético (2).

Os mecanismos fisiopatológicos que resultam na disfunção endotelial e a microangiopatia diabética vêm sendo elucidados ao longo do tempo. Dados recentes sugerem que a disfunção microcirculatória pode não estar relacionada a alterações da glicemia e anteceder em muitos anos o aparecimento do próprio diabetes $(3)$. Todavia, muitas questões ainda precisam ser respondidas. Por exemplo, não é conhecido o impacto da microangiopatia sobre a evolução de pacientes com doença arterial obstrutiva periférica (DAOP).

Os processos envolvidos na fisiopatologia da doença macrovascular se confundem com os da própria disfunção microcirculatória, e têm origem nos danos endotelial e da vasa-vasorum dos troncos arteriais, desencadeando o

\section{editorial}

\section{Carlos E. Virgini-Magalhães \\ ELIETE BOUSKELA}

Professor Adjunto e

Coordenador da Disciplina de

Cirurgia Vascular e

Endovascular do Hospital

Universitário Pedro Ernesto -

Universidade do Estado do Rio

de Janeiro (CEVM).

Professora Titular e

Coordenadora do Laboratório

de Pesquisas Clínicas e

Experimentais em Biologia

Vascular - BioVasc - Centro

Biomédico, Universidade do

Estado do Rio de Janeiro (EB),

Rio de Janeiro, RJ, Brasil. 
processo aterosclerótico e a DAOP. A DAOP caracteriza-se pela obstrução aterosclerótica progressiva das artérias dos membros inferiores afetando gradualmente, de forma adversa, a qualidade de vida de pacientes diabéticos e não-diabéticos. Em indivíduos diabéticos, as lesões ateroscleróticas apresentam algumas peculiaridades: iniciam mais precocemente, localizam-se especialmente nos troncos arteriais infrapatelares e preservam as artérias podais. As paredes arteriais em diabéticos são mais calcificadas e a calcificação da camada média (esclerose de Monckeberg) é freqüentemente observada em radiografias simples dos membros inferiores. As razões dessas diferenças não são conhecidas e suas conseqüências ainda precisam ser determinadas de forma efetiva.

Em nenhum outro território, as alterações das macro e microcirculações parecem atuar de forma tão sinérgica em detrimento do paciente como nos membros inferiores. A disfunção microcirculatória tende a amplificar os efeitos da macroangiopatia dos troncos arteriais (4). Aparentemente, o padrão da DAOP encontrado em diabéticos é mais grave e limita a possibilidade de realizar cirurgias de revascularização pela existência de doença difusa encontrada nas artérias infrapatelares. Quando a cirurgia de revascularização é factível, apresenta taxas de salvamento de membro semelhantes aos procedimentos realizados em indivíduos não-diabéticos, embora a mortalidade cirúrgica seja maior na população diabética (5). As alterações da microcirculação não são levadas em conta na decisão nem na estratégia cirúrgica e, no entanto, a disfunção microcirculatória pode explicar, pelo menos parcialmente, a deterioração de lesões que inicialmente parecem cicatrizar adequadamente após a revascularização, mas que evoluem para amputação do membro, a despeito do sucesso técnico do procedimento. Em outros tipos de intervenções, como em angioplastias, especialmente angioplastias subintimais, há evidências de que diabéticos apresentam maiores taxas de reestenoses (6).

A interação da doença vascular, da infecção e em especial da neuropatia periférica transforma o pé diabético em um órgão-alvo de altíssimo risco. Quinze por cento de indivíduos diabéticos desenvolverão ulceração dos pés em algum momento de suas vidas e, portanto, ficarão expostos à possibilidade de amputação de membros inferiores. O resultado é uma tragédia anunciada, e em nosso meio um grave problema de saúde pública. A cada 30 segundos um membro inferior é amputado ao redor do mundo. Aproximadamente $70 \%$ das amputações realizadas estão relacionadas ao diabetes e implicam taxas de mortalidade relativamente altas.

Simultaneamente à expansão do conhecimento mais profundo dos processos moleculares que envolvem a disfunção endotelial e microcirculatória, a última década foi prodigiosa em acumular sólidas evidências de que seja possível reduzir de forma significativa as taxas de amputação de membros inferiores com a ampla adoção de duas medidas: 1) o emprego de equipes multidisciplinares que incluam médicos de diferentes especialidades, podólogos, enfermeiras, nutricionistas e assistentes sociais no acompanhamento do pé de risco; e 2) educação. A educação tem sido identificada como o fator-chave na estratégia de melhorar o cuidado com os pés diabéticos (7). Nos dias de hoje, é possível afirmar que até $85 \%$ das amputações poderiam ser evitadas através de um programa de cuidado ao pé diabético bem organizado, controle glicêmico adequado, educação e informação.

Infelizmente, ainda estamos longe de reproduzir em larga escala o conhecimento adquirido, e o desfecho do pé diabético nos dias de hoje ainda não é favorável como poderia ser. A situação é grave e necessita mobilização de todos. Ainda existem muitas barreiras: pouca atenção governamental, restrições orçamentárias, ignorância e preconceito por parte de pacientes, familiares e equipe de saúde (8). A expansão do conhecimento científico estrito é apenas um desses obstáculos a serem vencidos.

\section{REFERÊNCIAS}

1. Rauner MS, Pesendrofer E. Model-Based evaluation of diabetic foot prevention strategies in Austria. Health Care Manag Sci. 2005;8:253-65.

2. Santos VP, Caffaro RA, Pozzan G, Saieg MA, Castelli Jr V. Comparative histological study of atherosclerotic lesions and microvascular changes in amputaded lower limbs of diabetic and non diabetic patients. Arq Bras Endocrinol Metab. 2008;52:1115-23.

3. Aguiar LGK, Villela NR, Bouskela E. A microcirculação no diabetes: implicações nas complicações crônicas e tratamento da doença. Arq Bras Endocrinol Metab. 2007;51:204-11.

4. Williams DT, Price P, Harding KG. The influence of diabetes and lower limb arterial disease on cutaneous foot perfusion. J Vasc Surg 2006;44:770-5.

5. Fratezi AC, Albers M, de Luccia ND, Pereira CA. Outcome and quality of life of patients with severe chronic limb ischaemia: a cohort study on the influence of diabetes. Eur J Vasc Endovasc Surg 1995;10:459-65.

6. Baumgartner I. In: Greenhalgh RM, editor. More Vascular and Endovascular Challenges. BIBA Publishing, London. 2007 
7. Papanas N, Maltezos E, Edmonds M. The diabetic foot: a plea for the elementary? Acta Diabetol 2006;43:152-3.

8. Rezende KF, Nunes MA, Melo NH, Mallerbi D, Chacra AR, Ferraz MB. Internações por pé diabético: comparação entre o custo direto estimado e o desembolso do SUS. Arq Bras Endocrinol Metab 2008;52:523-30.
Endereço para correspondência:

Carlos Eduardo Virgini-Magalhães

Boulevard 28 de Setembro 77, Vila Isabel

20551-030 - Rio de Janeiro, RJ

E-mail: virgini@uerj.br 